Research Article

\title{
Discrimination of Melanoma Using Laser-Induced Breakdown Spectroscopy Conducted on Human Tissue Samples
}

\author{
Muhammad Nouman Khan, Qianqian Wang $\mathbb{B}^{D}$, Bushra Sana Idrees, Geer Teng, Xutai Cui, \\ and Kai Wei
}

School of Optics and Photonics, Beijing Institute of Technology, Beijing 100081, China

Correspondence should be addressed to Qianqian Wang; qqwang@bit.edu.cn

Received 24 July 2020; Revised 23 November 2020; Accepted 18 December 2020; Published 30 December 2020

Academic Editor: Jau-Wern Chiou

Copyright (c) 2020 Muhammad Nouman Khan et al. This is an open access article distributed under the Creative Commons Attribution License, which permits unrestricted use, distribution, and reproduction in any medium, provided the original work is properly cited.

\begin{abstract}
Discrimination and identification of melanoma (a kind of skin cancer) by using laser-induced breakdown spectroscopy (LIBS) combined with chemometrics methods are reported. The human melanoma and normal tissues are used in the form of formalinfixed paraffin-embedded (FFPE) blocks as samples. The results demonstrated higher LIBS signal intensities of phosphorus (P), potassium $(\mathrm{K})$, sodium $(\mathrm{Na})$, magnesium $(\mathrm{Mg})$, and calcium $(\mathrm{Ca})$ in melanoma FFPE samples while lower signal intensities in normal FFPE tissue samples. Chemometric methods, artificial neural network (ANN), linear discriminant analysis (LDA), quadratic discriminant analysis (QDA), and partial least square discriminant analysis (PLS-DA) are used to build the classification models. Different preprocessing methods, standard normal variate (SNV), mean-centering, normalization by total area, and autoscaling, were compared. A good performance of the model (sensitivity, specificity, and accuracy) for melanoma and normal FFPE tissues has been achieved by the ANN and PLS-DA models (all were 100\%). The results revealed that LIBS combined with chemometric methods for detection and discrimination of human malignancies is a reliable, accurate, and precise technique.
\end{abstract}

\section{Introduction}

Cancer is an increasing concern worldwide and has become a major threat to human health. The leading causes of death are still due to various types of cancers. Skin cancer is one of the most frequently diagnosed types of cancer worldwide. For the identification and diagnosis of melanoma skin cancer analytical methods such as synchrotron radiation microanalysis (SXRF) [1-3], or transmission electron microscopy combined with energy dispersive $\mathrm{X}$-ray analysis (TEM-EDX) $[4,5]$, laser ablation inductively coupled plasma spectrometry, mass spectrometry (LA-ICP-MS) [5-8], and Raman Spectroscopy are already used [9]. In terms of LIBS sensitivity and spatial resolution with other techniques such as LA-ICP-MS, TEM, Nano-SIM (nanosecondary ion mass spectrometry), EPMA (electron probe micro-analysis), TEM, and SEM (scanning electron microscope), as indicated in [1], which illustrated that LIBS achieves micrometer scale resolution with part-per-million range sensitivities. The
LIBS detection range is much more than other techniques. In brief, comparison of LIBS feature that LIBS is better than $\mathrm{XRF}$ is as follows: for example, elemental detection range, isotope detection, spectral line interference, lateral spatial resolution, spatial resolution of depth, surface contamination sensitivity, rapidity of analysis, and safety during use [3]. Micro-SXRF is ideal for elemental mapping and chemical speciation with a spatial resolution down to less than $100 \mathrm{~nm}$ and a detection limit in the order of less than parts per million because of its high brightness and linear polarized existence [5].The sensitivity and resolution given by SXFR or microXRF is very high and is primarily used for $\mathrm{Cu}, \mathrm{Fe}$, and $\mathrm{Zn}$ imaging. Quantification with SXRF, however, remains difficult and appropriate calibration requirements should be achieved in an appropriate matrix [6,7]. LIBS has been the only all-optical technique that is entirely consistent with optical microscopy, providing part-per-million-range sensitivity (accessible for most metals) and micrometer-scale resolution [8]. The acquisition rate can be very high in LIBS 
since it is constrained only by the speed of the detector and the frequency rate of the laser. A pace of acquisition up to kilohertz has already been seen [9]. Although these analytical approaches showed high performance in terms of spatial resolution and sensitivity, their relatively slow analysis and the complexity of the required equipment make their use rather restrictive and difficult for routine medical diagnosis. Therefore, it is important to develop a precise and accurate method to identify and differentiate the melanoma samples from the normal.

In recent years, laser-induced breakdown spectroscopy (LIBS) has become a well-recognized and valuable analytical spectroscopic technique for the identification and analyses of different tissues with the ability to detect all the elements on the periodic table, including low- and high- $Z$ elements [10-12]. Some additional advantages of LIBS are acknowledged such as its ability to work at room temperature and atmospheric pressure, ease of use, simplicity in apparatus, full compatibility with optical microscopy, and the prospect of standoff and on-site operations, and there is no sample size or shape restrictions. In LIBS, the laser pulse is focused on the sample surface. Plasma can be formed if the irradiance at the focal spot overreach a specific threshold valued. Excitation and deexcitation of ions and atoms in the plasma of the ablated sample will give out fingerprint emission of the different elements. The chemical components of the samples can be firmed by analysing atomic and ionic emissions, and the sample can then be discriminated and classified [13].

In the literature, LIBS has been widely used to investigate various types of malignancies discrimination. Kumar et al. used LIBS spectra intensities difference ratios of various elements of the histological sections of dog liver, to distinguish normal liver and hemangiosarcoma [14]. Han et al. used LIBS spectra in the presence of argon flow rate $=15 \mathrm{~L} /$ min of mice sample (in the form of tissue and pellets) for a distinction of surrounding dermis and melanoma via LDA model for quantitative analysis difference of magnesium and calcium [13]. Gaudiuso et al. used mice blood and tissues as a sample, for the early diagnosis of melanoma; they obtained an accuracy of up to $96 \%$ [15]. To the best of our knowledge, here we, for the first time, evaluated the use of LIBS to obtain elemental analyses, the classification and discrimination of paraffin-embedded human biopsies of healthy and malignant skin tissues combined with chemometric methods.

In the present work, LIBS combined with chemometrics approaches has been used for investigations and discriminations of human melanoma FFPE tissue samples instead of animal samples. We performed our experiment in an open environment while using no inert gasses during sample ablation in experiments. We also investigate the performance of the model for different types of input data (10 lines intensities, 27 lines intensities, and all lines intensities). For precise and quantified results, data preprocessing plays a vital role. The analyst uses different procedures for data analysis. The main difference in the results can come from the use of different algorithms to perform deionizing and/or baseline subtraction $[16,17]$, to extract the emission signals for the selection of spectral line analysis. We also proposed different preprocessing methods like standard normal

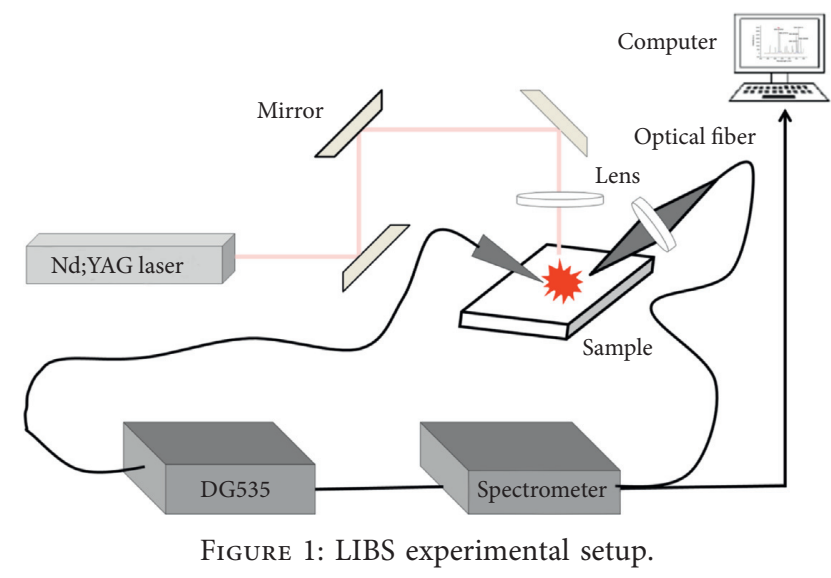

variate (SNV), autoscaling (auto), mean-centering (mean), and normalization by area (norm). We investigated which preprocessing method performed better and which data set line intensity is suitable in ANN, QDA, LDA, and PLS-DA models by comparing their impacts on the final classification accuracies, respectively. The performances of the models are determined in terms of accuracy, sensitivity, and specificity along with suitable preprocessing methods, and also reported P (phosphorus) line of the FFPE tissue samples, while the above-reported results about melanoma references $[13,15]$ did not report any $\mathrm{P}$ lines in their spectra because of mice samples (tissue, pellets, and blood) and early melanoma diagnosis. Based on these results, we analysed distinguishable elements from melanoma FFPE tissues, reflecting the clinical situation.

\section{Experimental Setup and Samples}

2.1. LIBS Experimental Setup. As shown in Figure 1, a flashpumped Q-switched Nd: YAG laser at $1064 \mathrm{~nm}$ with pulse duration $5 \mathrm{~ns}$, repetition frequency $1 \mathrm{~Hz}$, energy $64 \mathrm{~mJ} /$ pulse, and beam diameter $\varnothing 6 \mathrm{~mm}$ was used as the excitation source. The laser pulse was focused on the sample surface by three mirrors and a convex lens with a $100 \mathrm{~mm}$ focal length. The fiber with a diameter $\varnothing 600 \mu \mathrm{m}$ was used to collect plasma emission by another convex lens with a $36 \mathrm{~mm}$ focal length. Two-channel spectrometer (AvaSpec 2048-2-USB2 and Avantes) was connected to the outlet of optical fiber. The samples were placed on a $3 \mathrm{D}$ translation stage in order to ablate the fresh tissue samples spots. The coverage range of the spectrometer was $190 \mathrm{~nm}-1100 \mathrm{~nm}$ with $0.2 \sim 0.3 \mathrm{~nm}$ resolution. A digital delayer (SRS-DG535, Stanford Research System) and a photodetector were also used in the system. When the laser signal is detected by a photodetector, the spectrometer was triggered by DG535 after a preset delay time, $1.28 \mu \mathrm{s}$ in our case, to reduce the bremsstrahlung radiation. The time of integration of CCD detector was set to $2 \mathrm{~ms}$. The experiment was performed in an open environment without any buffer gasses.

2.2. Samples. The melanoma and normal FFPE tissues were used as the samples in the study; some images of samples are shown in Figure 2 before and after laser ablation. The 


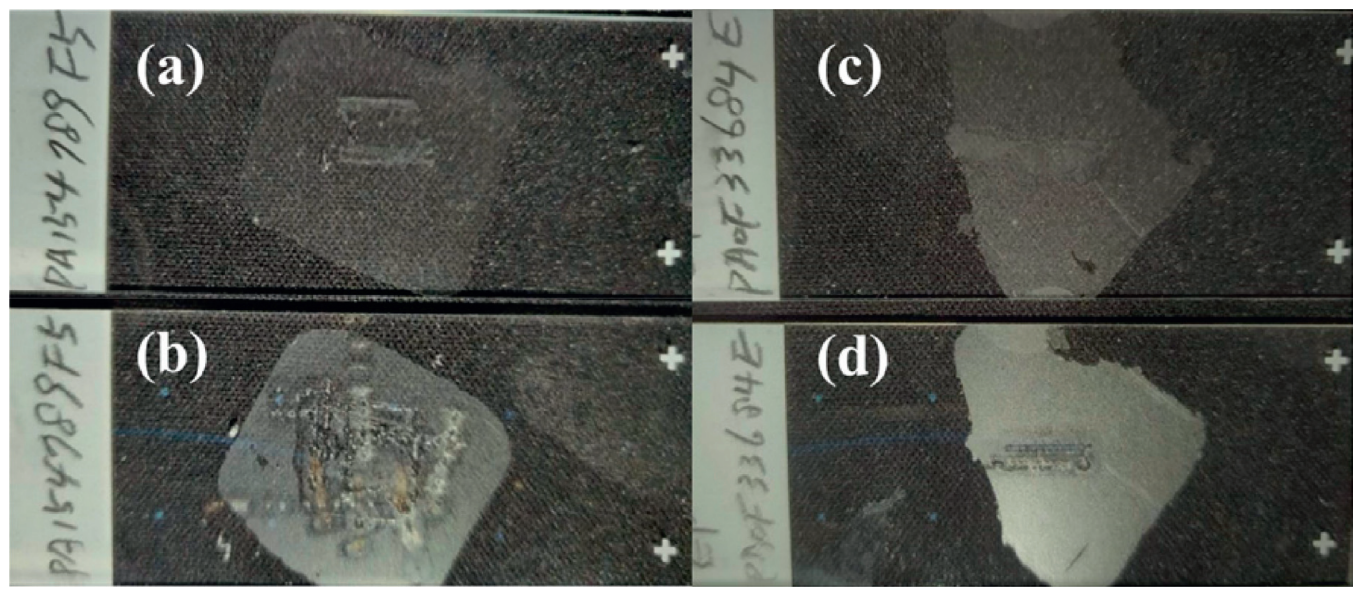

FIgURE 2: The images of FFPE normal and melanoma tissues on glass slides, before and after laser ablation: (a) normal FFPE tissue before laser ablations; (b) after laser ablation; (c) melanoma FFPE tissue before laser ablations; (d) after laser ablation, also laser-ablated seen.

samples were collected from the Origene Technologies Inc. of America with ethical permission from the Chinese custom Authority.

Based on the anatomic level of invasion of the layers of skin and vertical thickness of the lesion in millimetres, two classification schemes have been developed for skin cancer. For accurate prediction of future tumour behaviour, the most exclusive classification, the Breslow classification scheme is used recently. The stage of thin (T1) melanoma was classified by Clark level from the last decades [18]. In our research, the normal FFPE samples were collected from stage I melanoma patients and the melanoma FFPE samples were from stage III $\mathrm{C}$ patients. The number of the normal and the melanoma spectra was 111 , respectively.

2.3. Data Preprocessing. Usually, due to interference of surrounding air, fluctuation of laser energy, and inhomogeneity of sample surface, spectral fluctuations are produced between the measurements of each pulse. To reduce data fluctuations, suitable preprocessing methods are needed [19]. For obtaining a reliable data matrix, proper preprocessing of the raw data is very important, to perform the actual statistical analysis. In addition, proper data preprocessing appreciably upgrade and clarify the data analysis [20-22]. Four types of preprocessing methods are used in the present study, that is, autoscaling, normalization by total area, mean-centering, and SNV. The scaling process gives equal importance to all data sets and should not be suitable for noisy (i.e., poor signal-to-noise ratio) data [22].

2.4. Classification Models. In the present study, four types of classification models (ANN, PLS-DA, LDA, and QDA) are used on the four types of different preprocessing methods (autoscaling, mean-centering, normalization by total area, and SNV). For ANN networks, feedforward networks are used because of their excellent ability of self-adapting and self-learning. The two common and principal network types of feedforward networks are radial basis function
(RBF) and multilayer perceptron (MLP). For data analysis when ANN is used, it is very essential to determine between the ANN model (the network's arrangement) and ANN algorithms (computation that eventually produces the network output). The network that has been constructed for an application is ready to be trained. There are two types of training approaches, which are supervised and unsupervised. A fully connected ANN is used most frequently, with a backpropagation learning rule supervised network. ANN model of this type is better for discrimination purposes [23].

PLS-DA is one of the supervised methods and needs a learning step prior to its application to unknown samples. The interclass difference increases while the intraclass difference decreases in the PLS-DA model, which revealed the model recognition ability. PLS-DA model is very suitable for binary classification, while complexity will increase in the case of multiclassification PLS-DA model [24]. PLS-DA has also been applied to the classification of LIBS data and discriminates the tissue samples [25].

For classification purposes, both LDA and QDA are also used and achieved good accuracy results [26, 27]. LDA and QDA models are derived from simple probabilistic models, which model the class conditional distribution of the data $P$ $(X \mid y=k)$ for each $k$ class. Bayes rules are used for predicting the class as shown in

$$
P(y=k \mid X)=\frac{P(X \mid y t=n k) P(y=k)}{\sum_{l} P(X \mid y t=n l) P(y=l)},
$$

where $X$ and $y$ are events, $P(y=k \mid X)$ is a conditional probability, and $k$ is the class which maximizes the conditional probability.

Sensitivity is the ability of a test which decides the disease cases correctly, while specificity is defined as the ability of a test which decides and determines the healthy cases. Similarly, accuracy is defined as the ability of a test to demarcate the disease and healthy cases precisely. Sensitivity, specificity, and accuracy are calculated by the following equations, respectively: 


$$
\begin{gathered}
\text { sensitivity }=\frac{\mathrm{TP}}{\mathrm{TP}+\mathrm{FN}} \times 100 \%, \\
\text { specificity }=\frac{\mathrm{TN}}{\mathrm{TN}+\mathrm{FN}} \times 100 \%, \\
\text { accuracy }=\frac{\mathrm{TP}+\mathrm{TN}}{\mathrm{TP}+\mathrm{TN}+\mathrm{FP}+\mathrm{FN}} \times 100 \%,
\end{gathered}
$$

where (TP) is true-positive, the number of cases precisely recognized as a disease, (FP) is false-positive, the number of cases incorrectly identified as a disease, (TN) is true-negative, the number of cases precisely recognized as healthy, and (FN) is false-negative, the number of cases incorrectly identified as healthy.

\section{Results and Discussion}

3.1. LIBS Spectra Measurement Results. The typical LIBS spectra of human melanoma and normal FFPE tissue samples are illustrated in Figure 3(a), which is the average of 111 spectra in the spectral range of $200-900 \mathrm{~nm}$ of each sample. The LIBS spectra are usually normalized for discrimination purposes to reduce the spectral fluctuations that are produced due to the matrix effects and experimental variations. The normalization is operated comparatively to a specific line. The Carbon C $247.482 \mathrm{~nm}$ line is used for normalization purposes because the $\mathrm{C}$ emission is a specific line [13]. Carbon C $247.482 \mathrm{~nm}$ is used for normalization purposes because the $\mathrm{C}$ emission is firmed among all the measurement of LIBS spectra. The normalized LIBS spectra of the samples in the spectral region of 200 to $900 \mathrm{~nm}$ are also illustrated in Figure 3(b). Emission lines of magnesium $\mathrm{Mg}$ II, Mg I, calcium Ca I, Ca II, iron Fe I, phosphorus PII, sodium $\mathrm{Na} I$, potassium $\mathrm{K} \mathrm{I}$, hydrogen $\mathrm{H}$, oxygen $\mathrm{OI}$, and CN molecular bands $(386-389 \mathrm{~nm})$ are observed in the melanoma and normal FFPE tissue samples spectra. The spectral intensities of the melanoma and normal FFPE tissue samples are different. For example, the intensities of Ca II $(393.366 \mathrm{~nm}$ and $396.847 \mathrm{~nm})$, Ca I $(422.67 \mathrm{~nm})$, P I $(531.607 \mathrm{~nm})$, and $\mathrm{K} \mathrm{I}(766.5 \mathrm{~nm}$ and $769.9 \mathrm{~nm})$ in melanoma FFPE tissue sample are stronger as compared to those in normal FFPE tissue sample. It is notable that the spectral data intensities and ratio between them are very important for the classification purpose of FFPE tissue samples.

For further classification analysis, these 27 element lines are selected as features variables of the LIBS spectra. The main spectral lines can be seen, which is located at the ionic and atomic lines from $\mathrm{Ca}, \mathrm{Fe}, \mathrm{H}, \mathrm{K}, \mathrm{Mg}, \mathrm{Na}, \mathrm{O}$, and $\mathrm{P}$. These atomic and ionic emission lines of melanoma are more vigorous than those of the normal FFPE tissue samples. Other emission lines like $\mathrm{Fe}$ and $\mathrm{H}$ are frail and weaker. Therefore, these emission lines are listed in Table 1 for the classification of FFPE melanoma and normal tissue sample.

We detected significant changes in the LIBS spectra between melanoma and normal samples. In melanoma FFPE tissue samples, the peak intensities of $\mathrm{Ca}, \mathrm{K}, \mathrm{Mg}$, and $\mathrm{P}$ increase relative to the normal FFPE tissue peak intensity. Magnesium plays an important role in regulating cell division as well as $\mathrm{Ca}$. The melanin-containing pigment granules have an enormous amount in melanoma cells, which are wealthy in $\mathrm{Ca}$ [28]. The high levels of $\mathrm{Ca}$ are also reported in previous literature in different cancer tissues, like breast cancer, colorectal cancer [13], uterine cancer [29], and canine hemangiosarcoma [14]. Magnesium has also an important role in cell proliferation and the biosynthesis of proteins [30]. In the LIBS imaging technique, the elemental images of the tumour reported a gradient concentration for $\mathrm{Ca}, \mathrm{Mg}$, and $\mathrm{P}$ in metastatic melanoma cases, and also reported the wide dynamic ranges of concentration for elements, such as Fe and $\mathrm{Na}$ from low to high concentration [31]. A bioelemental study of melanoma lymph nodes also showed a clear distinction of $\mathrm{P}$ from the normal tissue [32].

The normalized intensities of 27 emission lines of the melanoma and normal FFPE tissue samples are shown in the bar graph in Figure 4. These 27 emission lines are selected as features emission lines of normal and melanoma spectra. Then, the area average was taken for these 27 elemental lines and selected only 10 lines on the basis of their normalized spectral intensities to draw a threshold line, which is also shown in Figure 4. For discrimination purposes, three types of input variables are used as input data for all the classification models (10 lines, 27 lines, and all lines intensities, resp.).

3.2. Classification Results. Classification accuracy is defined as the division of the number of correct predictions by predictions number, multiplied by $100 \%$ to gain the percentage accuracy. We performed all the models with the training set including 83 spectra of melanoma and normal sample (166 spectra in all) and a test set including 27 spectra of melanoma and normal sample (54 spectra in all).

For the ANN model, the number of hidden layers of neurons $l$, which needs to be optimal, is calculated according to the formula

$$
l=\sqrt{n+m}+\alpha
$$

The accuracy of the ANN model for different input variable data sets (10 lines, 27 lines, and all lines) is illustrated in Figure 5. In 10-line input variable data set, the highest accuracy is $100 \%$ for an autoscaling method in all hidden layers of a neuron, while the mean-centering, normalization by total area, and SNV preprocessed method showed $98 \%$ and 97\%, respectively, which is shown in Figure 5(a). Similarly, for 27-line intensity, input variables data set mean-centering showed the highest accuracy $100 \%$, while the autoscaling and SNV showed 98\% accuracy for different hidden neurons' layers as shown in Figure 5(b). Furthermore, for the whole input spectra variable data set, the ANN accuracy is $98 \%$ in autoscaling and $99 \%$ for normalization in all hidden layers, while the mean-centering and SNV showed very low accuracy for all the hidden layers as shown in Figure 5(c). Overall, the normalization data set revealed the best sensitivity, specificity, and accuracy results, for the ANN model. So, for tissue analysis, the normalization by area is the best and suitable compared to other preprocessing 


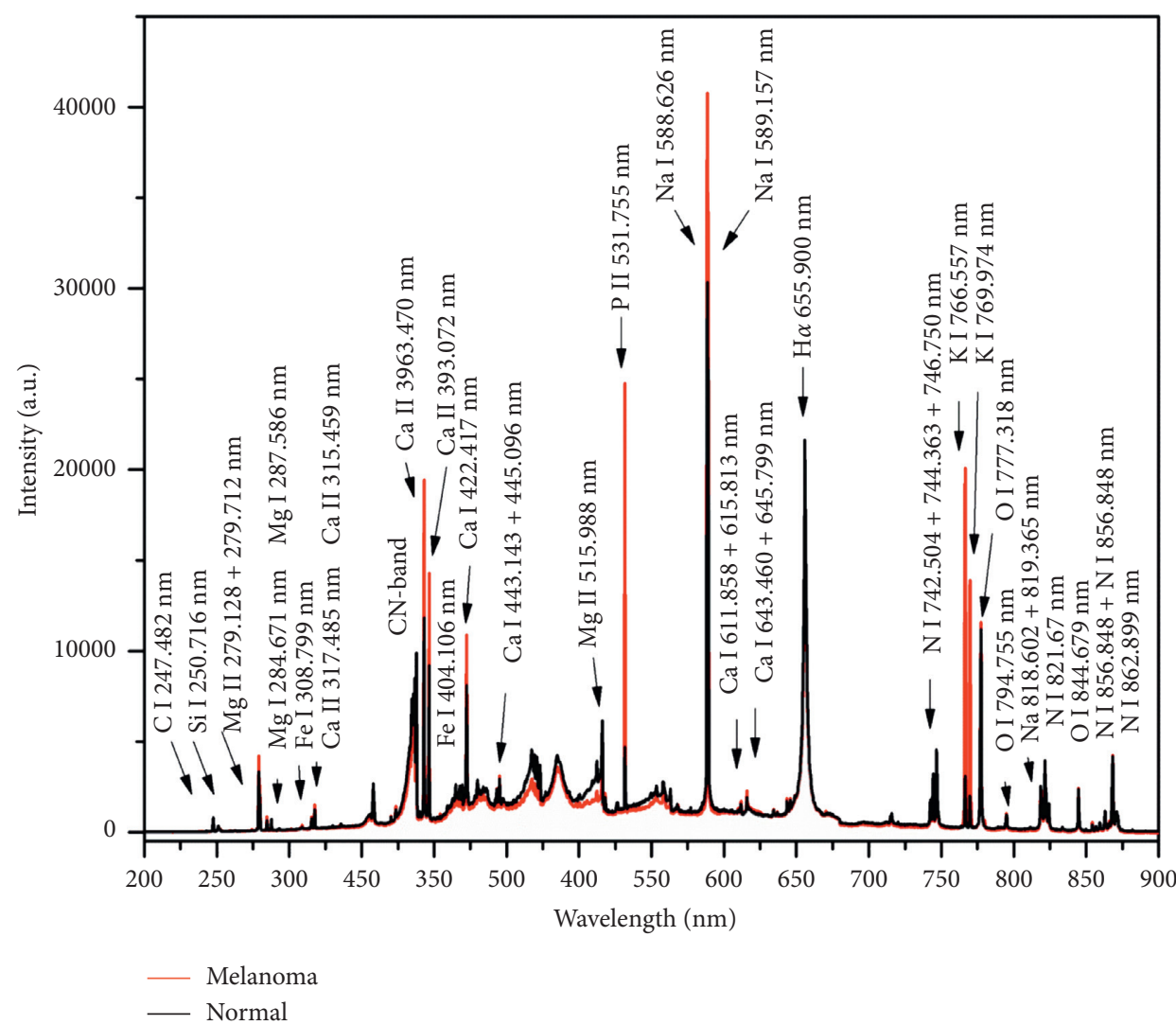

(a)

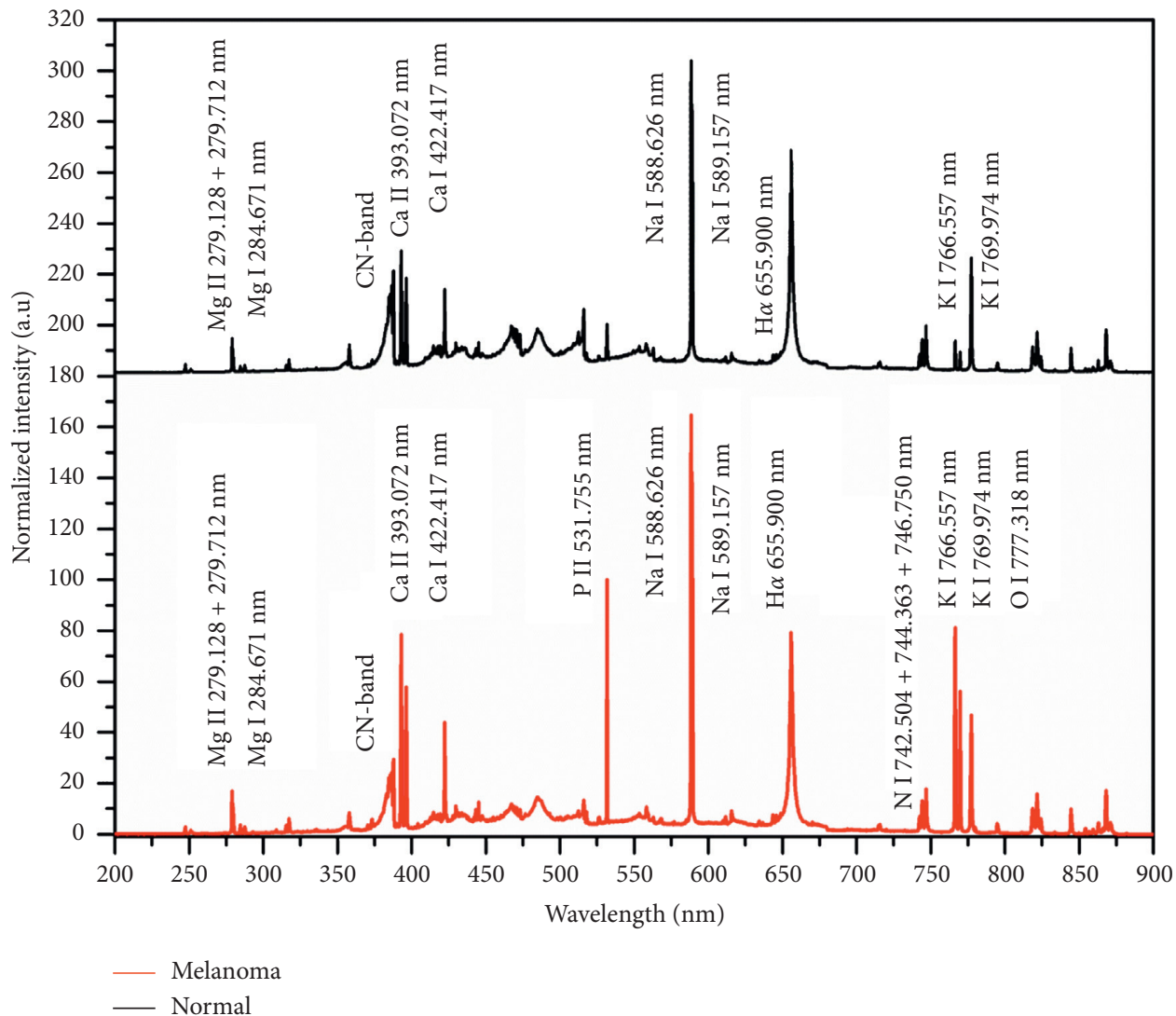

(b)

FIGURE 3: LIBS spectra of human melanoma and normal FFPE tissue samples (average of 111 measurement points of each sample) in the spectral range of 200-900 nm. (a) The original and (b) normalized spectra of the FFPE sample of melanoma and normal tissue, respectively. 
TABLE 1: The selected atomic and ionic emission lines.

\begin{tabular}{lc}
\hline Elements & Wavelength $(\mathrm{nm})$ \\
\hline Mg II & $279.552,280.270,515.762$ \\
Mg I & $285.6,287.586$ \\
Ca I & $422.67,443.142,445.096,611.873,615.813$ \\
Ca II & $315.459,317.483,370.392,373.466,393.366,396.847$ \\
Fe I & $308.798,358.046,404.106$, \\
P II & 531.607 \\
Na I & $588.99,589.59,819.48$ \\
K I & $766.5,769.9$ \\
H $\alpha$ & 656.270 \\
O I & 777.318 \\
\hline
\end{tabular}

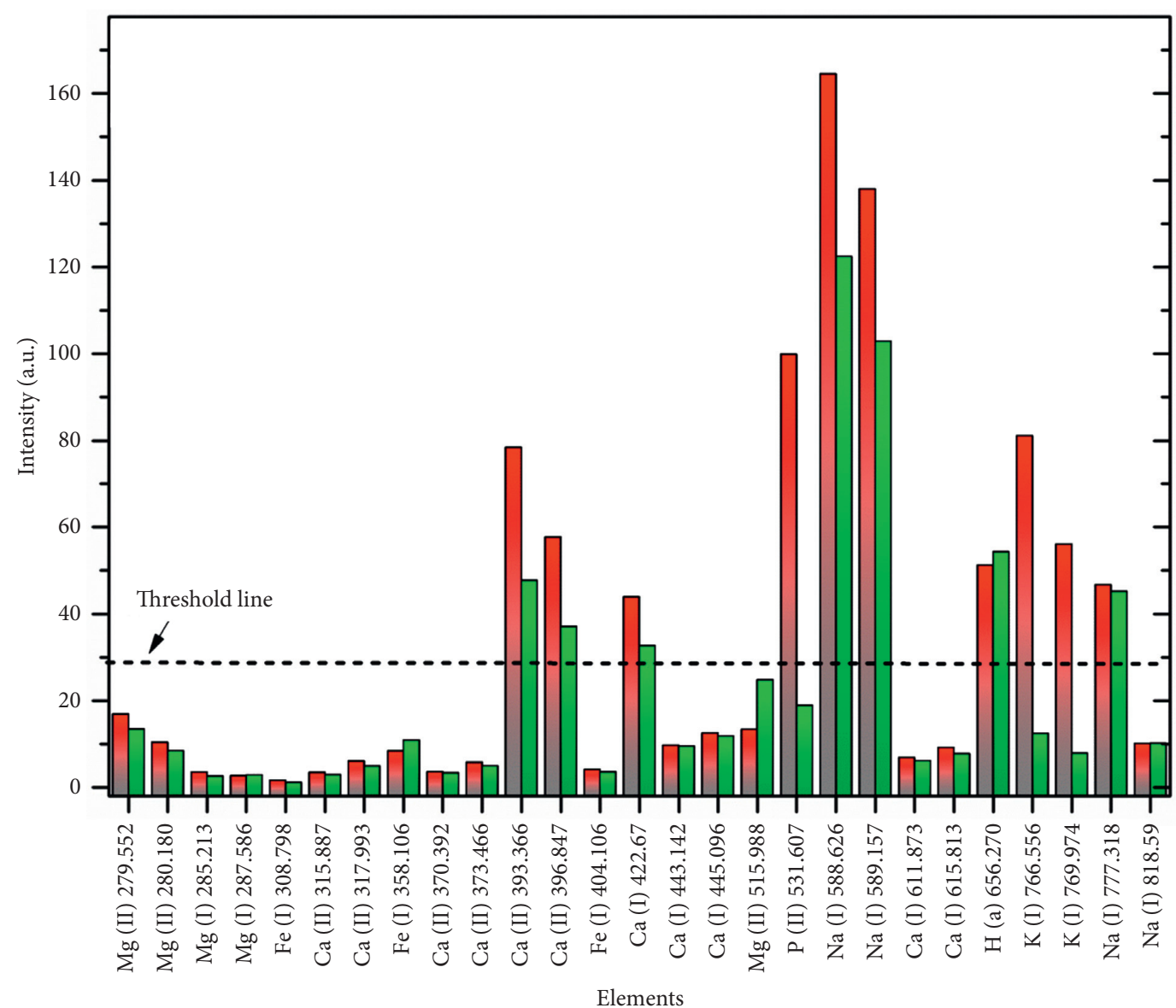

Melanoma

Normal

FIgURE 4: Evaluation of the normalized intensities of 27 LIBS emission lines of human FFPE samples of the melanoma and healthy tissue.

methods in the ANN model; the accuracy \% result of the ANN model is illustrated in Figure 6(a).

PLS-DA is also used to discriminate against the tissue samples. The 10-line intensity data set showed the highest accuracy result for PLS-DA model classification. Both meancentering and SNV data sets showed good classification accuracy as compared to other preprocessing data sets as revealed in Table 2. The classification result of the PLS-DA model for a different preprocessing method is shown in Figure 6(b).
For classification purposes, both LDA and QDA are also used and achieved good accuracy results. In the LDA model, the accuracy of classification for all the cases (10 lines, 27 lines, and all lines) cannot achieve the $100 \%$ for FFPE tissues differentiation. The highest and lowest accuracy values for LDA classification model are $98 \%$ and $77 \%$, respectively. While in QDA model classification, normalization by total area preprocessed method achieved 98\% accuracy in the 27 line input variable data set. The minimum accuracy for QDA model classification is calculated for the whole spectra input 


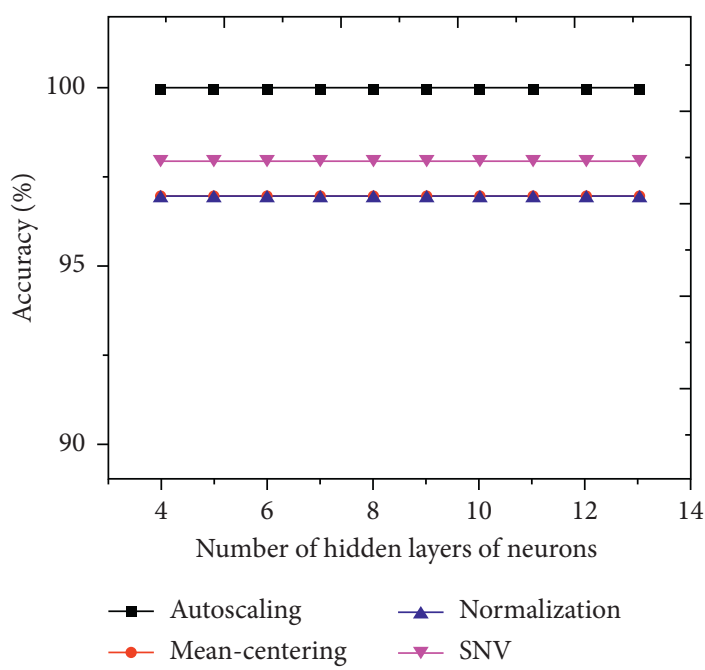

(a)

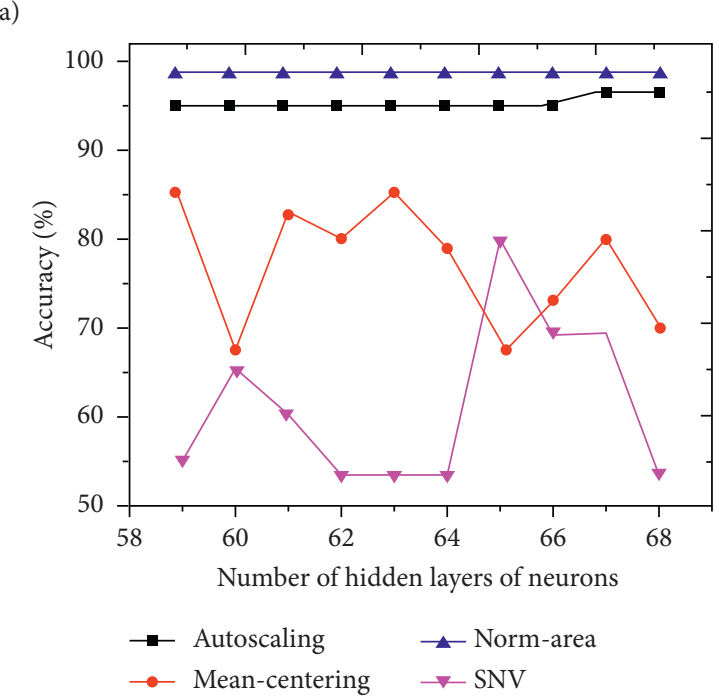

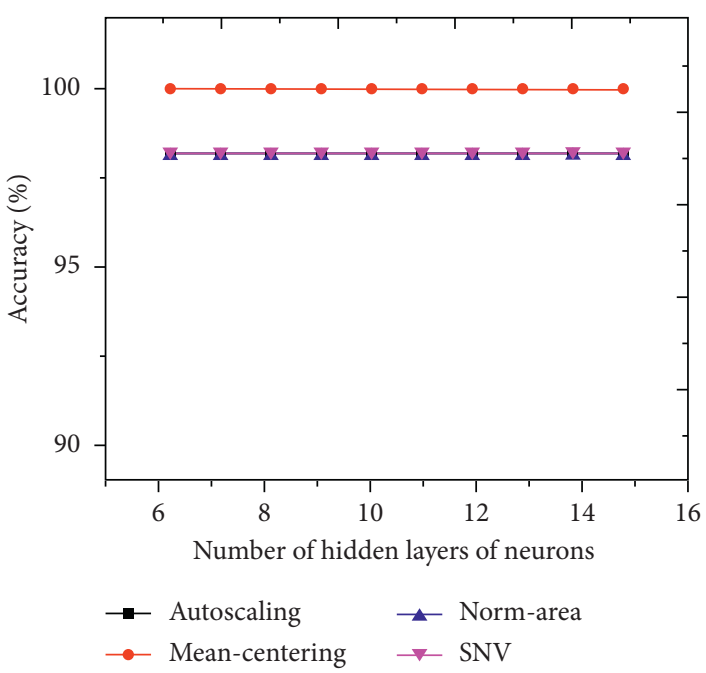

(b)

(c)

FIGURE 5: Accuracy results of ANN model generated by different types of input data: (a) the 10-line intensity, (b) 27-line intensity, and (c) all lines intensities, by using different preprocessing methods.

variable data set in SNV preprocessed method, which is $77 \%$. The percentage accuracy rate of LDA and QDA models are shown in Figures 6(c), and 6(d), respectively.

The accuracy results of ANN, PLS-DA, LDA, and QDA models along with suitable and appropriate preprocessing methods are illustrated in Table 2, which showed the highest accuracy for different input variable data sets and suitable preprocessed method. In the ANN model for the 10-line input variable data set, only the autoscaling preprocessed method showed the highest $100 \%$ accuracy, while for the 27 line input variable data set only the mean-centering showed the highest $100 \%$ accuracy. For the whole spectral input data set, only the norm-area preprocessing method showed the highest $98 \%$ accuracy, while the other preprocessed methods showed lower accuracy results. Similarly, in the PLS-DA model for 10-line intensity input variable data set, three preprocessing methods (autoscaling, SNV, and mean-centering) showed the highest $100 \%$ accuracy; on the other hand, for 27-line intensity and whole spectral data set, the preprocessed methods (mean-centering, normalized by the area, and SNV) showed the highest $100 \%$ accuracy, respectively. Furthermore, the LDA model classification for 10-line input variable data set showed the highest $98 \%$ accuracy for the autoscaling preprocessed method, while for 27-line intensity, the only normalization by area (normarea) revealed $98 \%$ accuracy result and for the whole input variable data set and also only the autoscaling preprocessed method showed 98\% accuracy result. In the QDA model classification for 10 lines, input variable data set, only autoscaling, and normalization preprocessed method showed the highest $94 \%$ accuracy; similarly, for 27 -line input variable data set, normalization by area showed $100 \%$ accuracy as well, and for whole data input variable, only autoscaling revealed the highest $98 \%$ accuracy result for melanoma FFPE and normal tissue differentiation. In comparison, ANN and PLS-DA is the best classification 


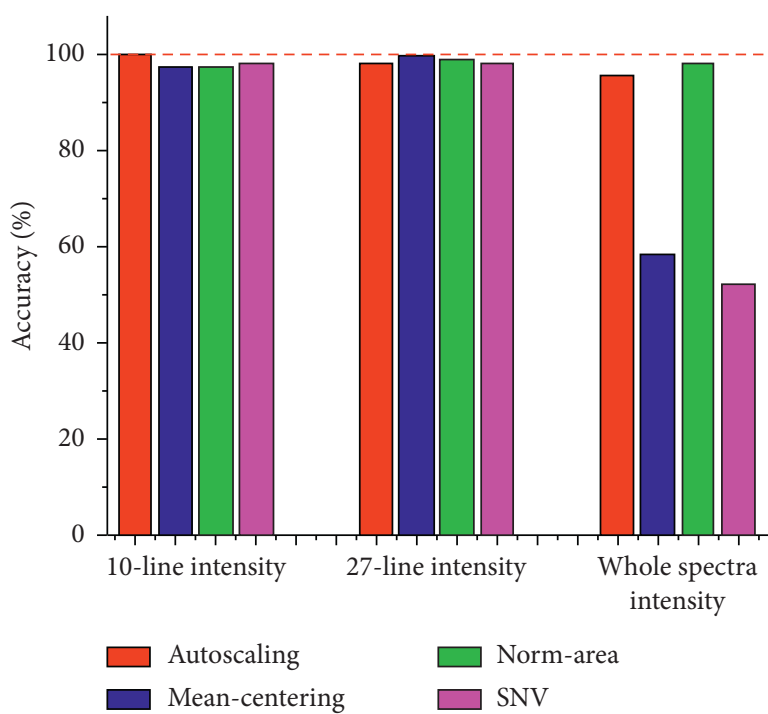

(a)

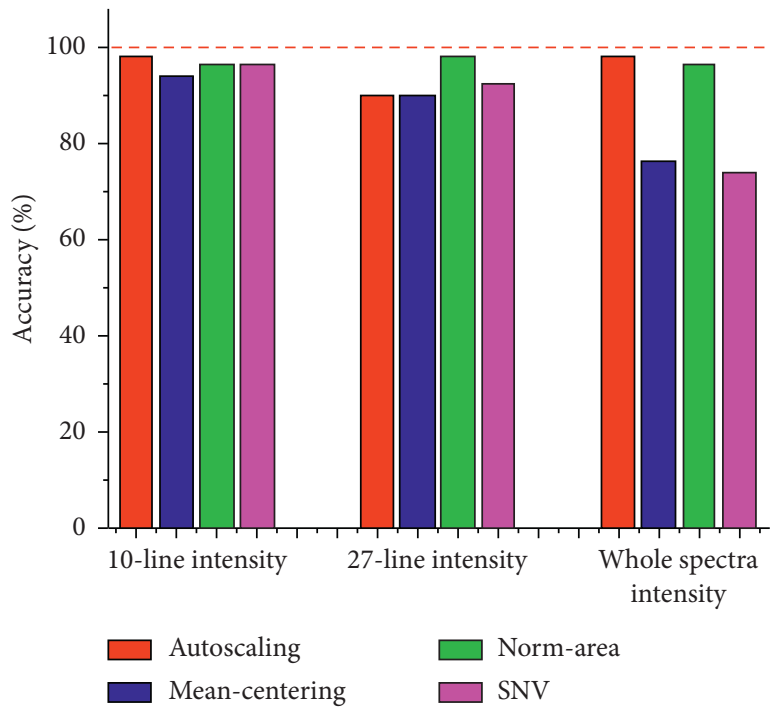

(c)

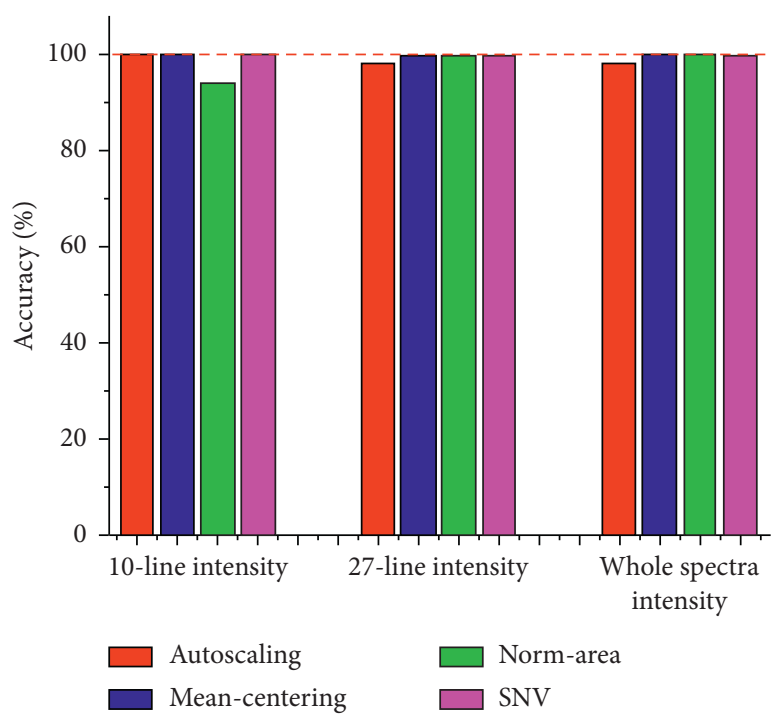

(b)

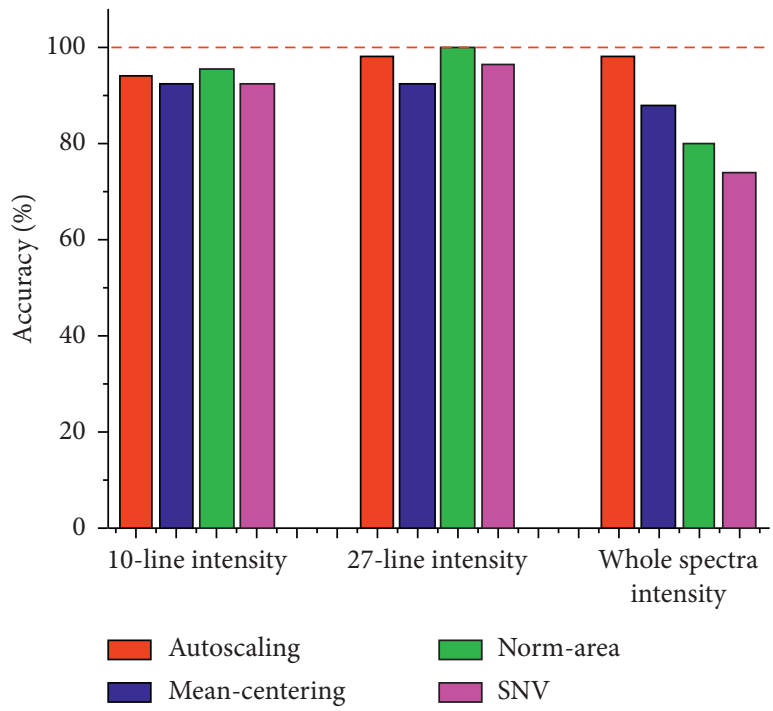

(d)

FIgure 6: The accuracy of (a) ANN model, (b) PLS-DA model, (c) LDA model, and (d) QDA model for different preprocessing methods and different types of input data (10-line intensity, 27-line intensity, and whole spectra).

model instead of QDA and LDA models for all types of input variable data sets.

Receiving operative curves (ROC) is a graphical plot that shows the diagnostic capability of a binary classifier system as its discrimination threshold is varied. The ROC curves for ANN, PLS-DA, LDA, and QDA are revealed in Figures 7(a)7(d) for a different input variable data set of 10 lines, 27 lines, and whole spectral data along with different preprocessing methods, respectively. The best ROC result is shown by the PLS-DA model as shown in Figure 7(b)

The ROC curves of the ANN, PLS-DA, LDA, and QDA model showed the lowest accuracy of $98 \%$ for the 10-line input variable dataset. Similarly, for the ROC curve for 27line input variable data set, the highest sensitivity $100 \%$, specificity $100 \%$, and accuracy $100 \%$ for ANN, PLS-DA, and QDA model are shown, while LDA showed the same result as for 10-line input variable data set. On the other hand, for the whole input variable data set, the highest sensitivity $100 \%$, specificity $100 \%$, and accuracy $100 \%$ are revealed for PLS-DA models while for LDA and QDA classification models, the whole input variable data set showed the highest sensitivity $96 \%$, specificity $100 \%$, and accuracy $98 \%$. It is concluded that the PLS-DA models are the best and useful for FFPE tissue classification as compared to LDA and QDA models.

The classification compact results of all the preprocessing methods, input variable data sets, and all four types of models ANN, PLS-DA, LDA, and QDA are shown in Figure 8. For ANN model classification, the highest accuracy is $100 \%$, shown by the autoscaling preprocessed method in 10 line input variable data set, and mean-centering in 27-line input variable data set, respectively, while the lowest 
TABLE 2: Optimal accuracy of different models along with suitable preprocessing methods.

\begin{tabular}{lccc}
\hline Models & Input data & Accuracy (\%) & Suitable preprocessing \\
\hline \multirow{2}{*}{ ANN } & 10 lines & 100 & Auto \\
& 27 lines & 100 & Mean \\
& All lines & 98 & Norm \\
\hline \multirow{3}{*}{ PLS-DA } & 10 lines & 100 & Auto, mean, SNV \\
& 27 lines & 100 & Mean, norm, SNV \\
& All lines & 100 & Mean, norm, SNV \\
\hline \multirow{3}{*}{ LDA } & 10 lines & 98 & Auto \\
& 27 lines & 98 & Norm \\
& All lines & 96 & Auto \\
QDA & 10 lines & 94 & Auto, norm \\
& 27 lines & 98 & Auto \\
\end{tabular}

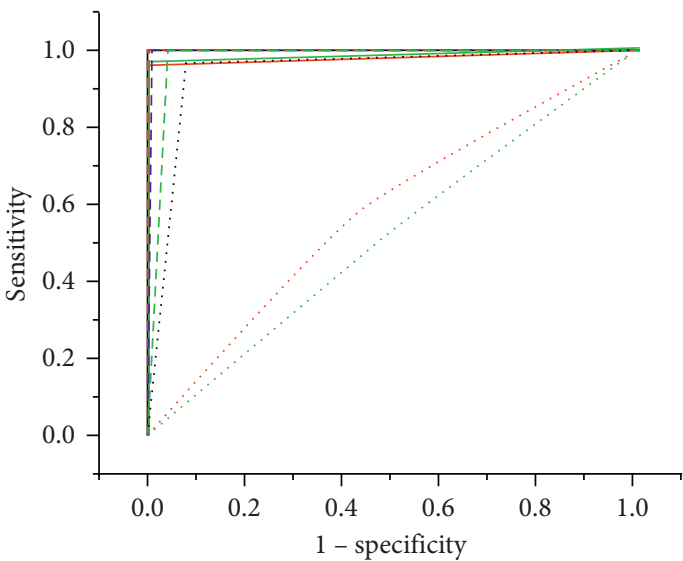

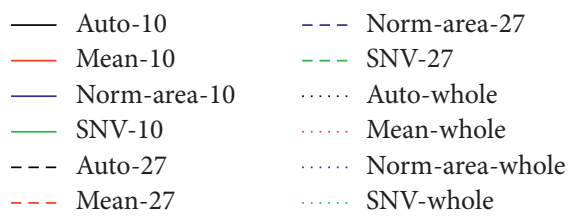

(a)

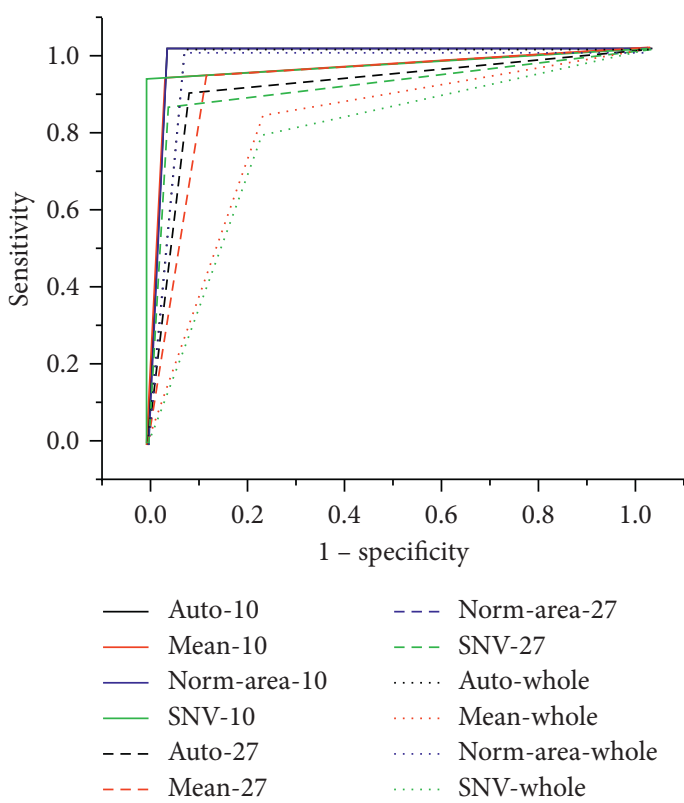

(c)

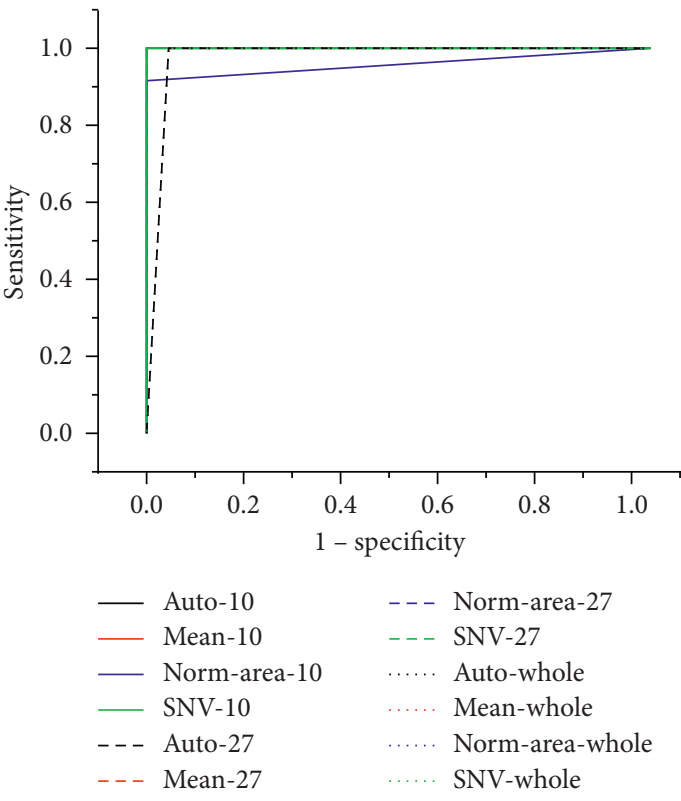

(b)

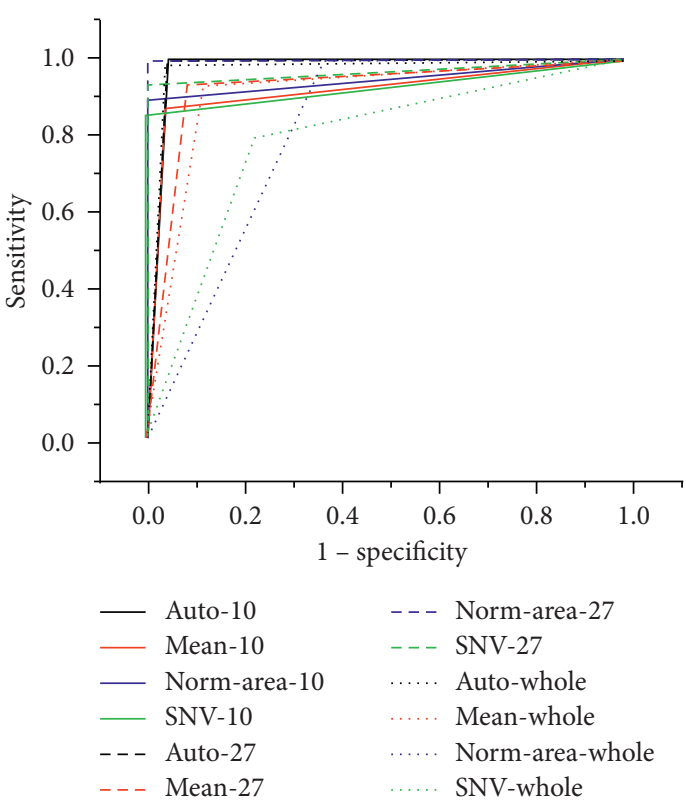

(d)

Figure 7: ROC curves of the (a) ANN model of 10-line input variable (solid line), 27-line (dash line), and the whole spectra (dot line) input data and different preprocessing methods; (b) PLS-DA; (c) LDA; and (d) QDA models showed the sensitivity and specificity, respectively. 


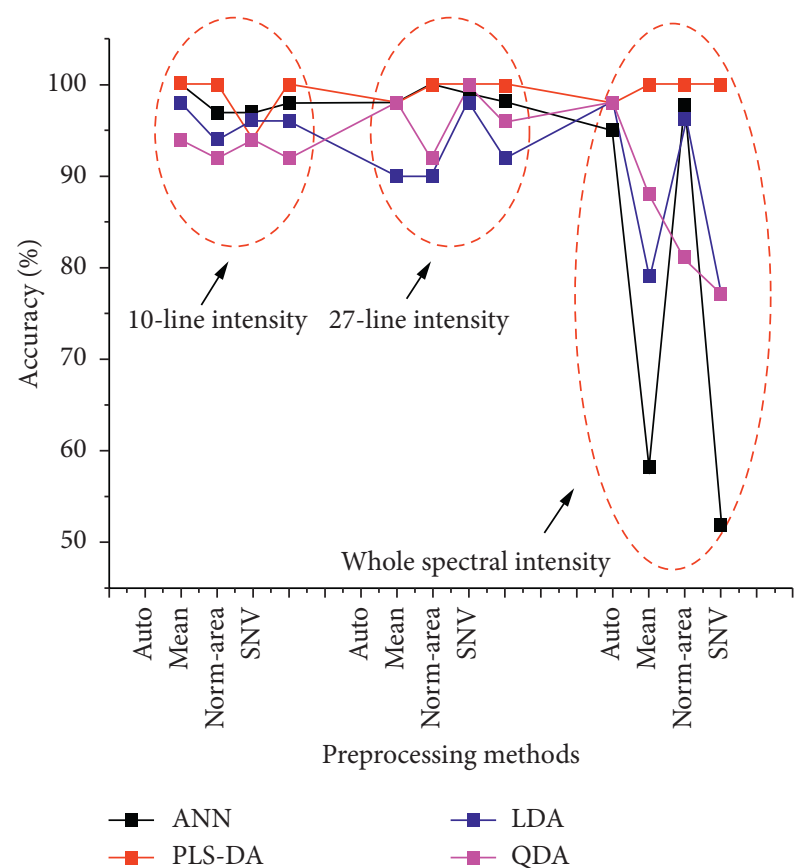

FIgURe 8: Accuracy of the ANN, PLS-DA, LDA, and QDA model by using different preprocessing methods for different types of input data.

(minimum) accuracy percentage is shown $52 \%$ by $\mathrm{SNV}$ preprocessed data in whole spectral input variable data set. Similarly, for PLS-DA model classification, the highest accuracy is $100 \%$, while the lowest is $94 \%$ for normalization by area (norm-area) in 10-line input variable data set. Furthermore, for the LDA model classification, the highest accuracy is $98 \%$ while the lowest (minimum) accuracy is $77 \%$ for SNV preprocessed method in the whole spectral input variable data set. On the other hand, for QDA classification model, the highest accuracy is $100 \%$, while the lowest is $77 \%$ for SNV preprocessed method in the whole spectral input variable data set, the same as the LDA model result for the whole input variable data set. All model results revealed that the 27 -line intensity was the average highest accuracy rate of $97.6 \%$ as compared to others; autoscaling data set showed the highest average accuracy $97.8 \%$ of all the models, and PLS-DA showed the averaged best performance model in all of them; average accuracy is $99.16 \%$.

\section{Conclusion}

The purpose of the research was to discriminate melanoma skin cancer and normal skin by using laser-induced breakdown spectroscopy (LIBS) combined with the chemometric methods. The melanoma and normal FFPE tissues were used as samples. In the LIBS spectra, the intensities of the lines of several elements showed a significant difference between the melanoma and normal FFPE samples and were regarded as feature variables. The lines intensities of calcium $(\mathrm{Ca})$, magnesium $(\mathrm{Mg})$, phosphorus $(\mathrm{P})$, potassium $(\mathrm{K})$, sodium $(\mathrm{Na})$, and oxygen $(\mathrm{O})$ in melanoma samples were higher than the normal samples. Phosphorus (P) line, especially, showed the highest intensity for malignancies. Therefore, phosphorus (P), calcium (Ca), magnesium (Mg), and potassium $(\mathrm{K})$ were defined as biomarkers for discrimination in this study. The chemometric models, such as ANN, PLS-DA, LDA, and QDA were used to analyse the spectral data from melanoma and normal tissue in FFPE. The best performance of the model (sensitivity, specificity, and accuracy) has been achieved by the ANN and PLS-DA models (all were 100\%). The results indicated that LIBS combined with the chemometric models could be used as a quick discrimination method for human malignancies.

\section{Data Availability}

The data used to support the findings of this study are available from the corresponding author upon request.

\section{Conflicts of Interest}

The authors declare that there are no conflicts of interest related to this article.

\section{Acknowledgments}

The research was supported by the National Natural Science Foundation of China (NSFC) under Grant 61775017 and Graduate Technological Innovation Project of Beijing Institute of Technology (2019CX20026).

\section{References}

[1] A. Sakdinawat and D. Attwood, "Nanoscale X-ray imaging," Nature Photonics, vol. 4, no. 12, pp. 840-848, 2010.

[2] W. Chao, B. D. Harteneck, J. A. Liddle, E. H. Anderson, and D. T. Attwood, "Soft X-ray microscopy at a spatial resolution better than 15 nm," Nature, vol. 435, no. 7046, pp. 1210-1213, 2005.

[3] W. Meyer-Ilse, D. Hamamoto, A. Nair et al., "High resolution protein localization using soft X-ray microscopy," Journal of Microscopy, vol. 201, no. 3, pp. 395-403, 2001.

[4] W. Rima, L. Sancey, M.-T. Aloy et al., "Internalization pathways into cancer cells of gadolinium-based radiosensitizing nanoparticles," Biomaterials, vol. 34, no. 1, pp. 181-195, 2013.

[5] T. Pluháček, K. Lemr, D. Ghosh, D. Milde, J. Novák, and V. Havlíček, "Characterization of microbial siderophores by mass spectrometry," Mass Spectrometry Reviews, vol. 35, no. 1, 2015.

[6] J. S. Becker, M. V. Zoriy, C. Pickhardt, N. Palomero-Gallagher, and K. Zilles, "Imaging of copper, zinc, and other elements in thin section of human brain samples (hippocampus) by laser ablation inductively coupled plasma mass spectrometry," Analytical Chemistry, vol. 77, no. 10, pp. 3208-3216, 2005.

[7] E. Moreno-Gordaliza, C. Giesen, A. Lázaro et al., "Elemental bioimaging in kidney by LA-ICP-MS as a tool to study nephrotoxicity and renal protective strategies in cisplatin therapies," Analytical Chemistry, vol. 83, no. 20, pp. 7933-7940, 2011.

[8] M. Bonta, J. J. Gonzalez, C. D. Quarles, R. E. Russo, B. Hegedus, and A. Limbeck, "Elemental mapping of biological samples by the combined use of LIBS and LA-ICP- 
MS," Journal of Analytical Atomic Spectrometry, vol. 31, no. 1, pp. 252-258, 2016.

[9] A. Seifalinezhad, M. Bahreini, M. M. Hassani Matin, and S. H. Tavassoli, "Feasibility study on discrimination of neoplastic and non-neoplastic gastric tissues using spark discharge assisted laser induced breakdown spectroscopy," Journal of Lasers in Medical Sciences, vol. 10, no. 1, pp. 64-69, 2018.

[10] L. Rinaldi, G. Barabino, J.-P. Klein et al., "Metals distribution in colorectal biopsies: new insight on the elemental fingerprint of tumour tissue," Digestive and Liver Disease, vol. 47, no. 7, pp. 602-607, 2015.

[11] A. Al-Ebraheem, E. Dao, K. Geraki, and M. J. Farquharson, "Emerging patterns in the distribution of trace elements in ovarian, invasive and in-situ breast cancer," Journal of Physics: Conference Series, vol. 499, 2014.

[12] U. Majewska, D. Banaś, J. Braziewicz, S. Góźdź, A. KubalaKukuś, and M. Kucharzewski, "Trace element concentration distributions in breast, lung and colon tissues," Physics in Medicine and Biology, vol. 52, no. 13, pp. 3895-3911, 2007.

[13] J. H. Han, Y. Moon, J. J. Lee, S. Choi, Y.-C. Kim, and S. Jeong, "Differentiation of cutaneous melanoma from surrounding skin using laser-induced breakdown spectroscopy," Biomedical Optics Express, vol. 7, no. 1, p. 57, 2016.

[14] A. Kumar, F.-Y. Yueh, J. P. Singh, and S. Burgess, "Characterization of malignant tissue cells by laser-induced breakdown spectroscopy," Applied Optics, vol. 43, no. 28, p. 5399, 2004.

[15] R. Gaudiuso, E. Ewusi-Annan, N. Melikechi et al., "Using LIBS to diagnose melanoma in biomedical fluids deposited on solid substrates: limits of direct spectral analysis and capability of machine learning," Spectrochimica Acta Part B: Atomic Spectroscopy, vol. 146, pp. 106-114, 2018.

[16] J. Peng, S. Peng, A. Jiang, J. Wei, C. Li, and J. Tan, "Asymmetric least squares for multiple spectra baseline correction," Analytica Chimica Acta, vol. 683, no. 1, pp. 63-68, 2010.

[17] M. D. Dyar, S. Giguere, C. Carey, and T. Boucher, "Comparison of baseline removal methods for laser-induced breakdown spectroscopy of geological samples," Spectrochimica Acta Part B: Atomic Spectroscopy, vol. 126, pp. 53-64, 2016.

[18] C. M. Balch, A. C. Buzaid, M. B. Atkins et al., "A new American Joint Committee on cancer staging system for cutaneous melanoma," Cancer, vol. 88, pp. 1484-1491, 2002.

[19] M. Zeaiter, J. M. Roger, and V. Bellon-Maurel, "Dynamic orthogonal projection. A new method to maintain the on-line robustness of multivariate calibrations. Application to NIRbased monitoring of wine fermentations," Chemometrics and Intelligent Laboratory Systems, vol. 80, no. 2, pp. 227-235, 2006.

[20] M. Zeaiter, J.-M. Roger, and V. Bellon-Maurel, "Robustness of models developed by multivariate calibration. Part II: the influence of pre-processing methods," TrAC Trends in Analytical Chemistry, vol. 24, no. 5, pp. 437-445, 2005.

[21] M. Zeaiter, J.-M. Roger, V. Bellon-Maurel, and D. N. Rutledge, "Robustness of models developed by multivariate calibration. Part I," TrAC Trends in Analytical Chemistry, vol. 23, no. 2, pp. 157-170, 2004.

[22] R. E. Millsap, A User's Guide to Principal Components, J. Edward Jackson, Ed., John Wiley \& Sons, New York, NY, USA, 1995.

[23] S. Agatonovic-Kustrin and R. Beresford, "Basic concepts of artificial neural network (ANN) modeling and its application in pharmaceutical research," Journal of Pharmaceutical and Biomedical Analysis, vol. 22, no. 5, pp. 717-727, 2000.

[24] N. C. Dingari, I. Barman, A. K. Myakalwar, S. P. Tewari, and M. Kumar Gundawar, "Incorporation of support vector machines in the LIBS toolbox for sensitive and robust classification amidst unexpected sample and system variability," Analytical Chemistry, vol. 84, no. 6, pp. 2686-2694, 2012.

[25] J. L. Gottfried, R. S. Harmon, F. C. De Lucia, and A. W. Miziolek, "Multivariate analysis of laser-induced breakdown spectroscopy chemical signatures for geomaterial classification," Spectrochimica Acta Part B: Atomic Spectroscopy, vol. 64, no. 10, pp. 1009-1019, 2009.

[26] F. C. De Lucia Jr., J. L. Gottfried, C. A. Munson, and A. W. Miziolek, "Multivariate analysis of standoff laser-induced breakdown spectroscopy spectra for classification of explosive-containing residues," Applied Optics, vol. 47, no. 31, p. G112, 2008.

[27] R. Kanawade, F. Mehari, C. Knipfer et al., "Pilot study of laser induced breakdown spectroscopy for tissue differentiation by monitoring the plume created during laser surgery - an approach on a feedback Laser control mechanism," Spectrochimica Acta Part B: Atomic Spectroscopy, vol. 87, pp. 175-181, 2013.

[28] A. El-Hussein, A. K. Kassem, H. Ismail, and M. A. Harith, "Exploiting LIBS as a spectrochemical analytical technique in diagnosis of some types of human malignancies," Talanta, vol. 82, pp. 495-501, 2010.

[29] S. Moncayo, J. D. Rosales, R. Izquierdo-Hornillos, J. Anzano, and J. O. Caceres, "Classification of red wine based on its Protected Designation of Origin (PDO) using Laser-induced Breakdown Spectroscopy (LIBS)," Talanta, vol. 158, pp. 185-191, 2016.

[30] W. D. Bush and J. D. Simon, "Quantification of $\mathrm{Ca}^{2+}$ binding to melanin supports the hypothesis that melanosomes serve a functional role in regulating calcium homeostasis," Pigment Cell Research, vol. 20, no. 2, 2007.

[31] M. Nasiadek, T. Krawczyk, and A. Sapota, "Tissue levels of cadmium and trace elements in patients with myoma and uterine cancer," Human \& Experimental Toxicology, vol. 24, no. 12, pp. 623-630, 2005.

[32] M. Terasaki and H. Rubin, "Evidence that intracellular magnesium is present in cells at a regulatory concentration for protein synthesis," Proceedings of the National Academy of Sciences of the United States of America, vol. 82, no. 21, pp. 7324-7326, 1985. 